\title{
ABUNDANCES OF YOUNG LMC CLUSTERS
}

\author{
R.A. Schommer \\ Rutgers University \\ Doug Geisler \\ CTIO/NOAO
}

ABSTRACT: We present Washington system photoelectric photometry for stars in three young LMC clusters: NGC 1850, NGC 1866, and NGC 2164. We derive metal abundances $[\mathrm{A} / \mathrm{H}]$ of $-0.3,-0.4$, and -0.6 , respectively.

\section{INTRODUCTION}

While the ages of several dozen Magellanic Cloud clusters have now been determined by main sequence photometry, few direct abundance determinations have been made in these clusters since the pioneering work of Cohen (1982). This is in part due to the faintness of the stars, which prohibits high dispersion abundance analyses, and the relatively high abundances, which are often beyond the calibrations of low dispersion techniques. We present here Washington photometry of individual stars in three young LMC clusters.

\section{THE DATA}

Photometry, using the CTIO 1.5 meter telescope, was performed in the Washington system on individual stars in NGC 1850, NGC 1866 and NGC 2164. The mean errors for one observation were 0.025 mag in both $M-T_{1}$, and $\mathrm{T}_{1}-\mathrm{T}_{2}$. These rather large errors arise from the varying background in the crowded LMC fields. For this reason the clusters chosen are far from the LMC bar, and brighter giants outside the cluster cores were selected.

Table I lists the observed colors for the stars. The $\Delta\left(M-T_{1}\right)$ colors were then calculated and the corresponding abundances were derived using a new calibration of the system discussed in Canterna et al. (1986) and Geisler (1986). The LMC clusters are somewhat younger than the Galactic counterparts detailed in these calibrations, so some effects due to the different surface gravities are possible, but these are expected to be small in $M-T_{1}$ (Canterna and Harris 1979). The ages for these clusters from Hodge (1983) are also given. 
TABLE I

\begin{tabular}{|c|c|c|c|c|c|c|c|c|c|}
\hline STAR & $\mathrm{M}-\mathrm{T}_{1}$ & $\mathrm{~T}_{1}-\mathrm{T}_{2}$ & $\Delta\left(\mathrm{M}-\mathrm{T}_{1}\right)$ & $\mathrm{n}$ & $E(B-V)$ & SWB & $\left\langle\Delta\left(M-T_{1}\right)\right\rangle$ & {$[\mathrm{A} / \mathrm{H}]$} & $t($ Gyr $)$ \\
\hline $1850-34^{\wedge} 1$ & 0.647 & 0.461 & 0.060 & 2 & 0.08 & II & 0.048 & -0.3 & 0.04 \\
\hline $1850-48$ & 0.944 & 0.614 & 0.035 & 2 & & & \pm .007 & \pm 0.1 & \\
\hline $1850-49$ & 0.799 & 0.542 & 0.050 & 2 & & & & & \\
\hline $1866-\mathrm{I}-02^{\wedge} 2$ & 0.665 & 0.445 & & 4 & 0.03 & III & 0.060 & -0.4 & 0.08 \\
\hline $1866-\mathrm{I}-03$ & 0.997 & 0.665 & 0.065 & 7 & & & \pm .009 & \pm 0.1 & \\
\hline 1866 -III - 09 & 0.747 & 0.532 & 0.099 & 3 & & & & & \\
\hline $1866-$ IV -10 & 0.747 & 0.495 & 0.045 & 6 & & & & & \\
\hline $1866-$ II - 13 & 0.767 & 0.571 & 0.159 & 1 & & & & & \\
\hline 1866 - III - 20 & 0.589 & 0.409 & 0.047 & 3 & & & & & \\
\hline 1866 -III - 22 & 0.920 & 0.610 & 0.068 & 4 & & & & & \\
\hline $2164-46^{\wedge} 3$ & 0.951 & 0.621 & 0.045 & 5 & 0.07 & III & I $\quad 0.084$ & -0.6 & 0.05 \\
\hline $2164-48$ & 0.753 & 0.527 & 0.086 & 7 & & & \pm 0.013 & \pm 0.2 & \\
\hline $2164-50$ & 0.766 & 0.571 & 0.103 & 5 & & & & & \\
\hline $2164-64$ & 0.858 & 0.586 & 0.100 & 3 & & & & & \\
\hline
\end{tabular}

1) Star identifications from Tifft and Connolly (1973), Table II SE.

2) Arp and Thackery (1967), inner annulus, except III-20, outer annulus

3) Star identifications in Hodge and Flower (1973).

\section{DISCUSSION}

The abundances are comparable with high dispersion analyses by Cohen (1982) for SWB type I-III clusters. We note that Nelles and Richtler (1984) derive a photometric abundance of -1.2 for NGC 1866, while Becker and Mathews (1983) analyzed the CMD of N1866 and found a solar metal abundance. It is premature to draw lengthy conclusions based upon the abundances in three LMC clusters. It appears that young LMC clusters are significantly metal-poor, by about 0.5 dex, compared with Galactic clusters of the same age (Canterna et al. 1986).

\section{REFERENCES}

Arp, H. C. and Thackeray, A.D. 1967 Astrophys. J., 149, 73.

Becker, S. A. and Mathews, G. J. 1983 Astrophys. J., 270, 155.

Canterna, R., Geisler, D., Harris, H., Olszewski, E.W. and Schommer, R.A. 1986 Astron. J., 92, 79.

Canterna, R. and Harris, H. C. 1979 in Problems of Calibration of Multicolor Photometric Systems, Dudley Obs. Rep. No. 14, A. G. D. Philip, ed., Dudley Obs., Schnectady, p. 199.

Cohen, J. G. 1982 Astrophys. J., 258, 143.

Geisler, D. 1986 Pub. Astron. Soc. Pacific, 98, in press.

Hodge, P. W. and Flower, P. J. 1973 Astrophys. J., 185, 829.

Hodge, P.W. 1983 Astrophys. J., 264, 470.

Nelles, B. and Richtler, T. 1984 in IAU Symposium No. 108, Structure and Evolution of the Magellanic Clouds, S. van den Bergh and K. S. de Boer, eds., Reidel, Dordrecht, p. 33.

Tifft, W. G. and Connolloy, L. 1973 Mon. Not. Roy. Astron. Soc., 163,93 . 